\title{
Etudes ethnomédicinale, phytochimie et activité antioxydante de Crateva adansonii $D C$ (Capparidaceae) dans les communes de Cotonou et de Dassa-Zoumè au Benin
}

Zinsou Franck Mignanwandé ${ }^{1}$, Armelle Sabine Yélignan Hounkpatin ${ }^{1,2}$, Roch Christian Johnson ${ }^{1}$, Delphin Anato ${ }^{1}$, Wilfrid Hinnoutondji Kpètèhoto ${ }^{1}$, Madjid Olatoundé Amoussa ${ }^{3}$

1 Université d'Abomey-Calavi, Centre Interfacultaire de Formation et de Recherche en Environnement pour le Développement Durable (CIFRED), Laboratoire d'Hygiène, d'Assainissement, de Toxicologie et de Santé Environnementale (HECOTES), 01 BP 1463, Cotonou, Bénin

${ }^{2}$ Université Nationale des Sciences, des Technologies, de l'Ingénierie et des Mathématiques (UNSTIM), École Normale Supérieure de l'Enseignants Technique (ENSET), BP 133 Lokossa, Bénin

3 Faculté des Sciences et Technologies, Laboratoire de Biochimie et de Substances Naturelles Bioactives (LBSNB)

*Auteur correspondant. Courriel: harmelle2011@gmail.com ; Tél: 00 (229) 97662855

Mots-clés : ethnomédicinale, Crateva adansonii; Phytochimie; Chromatographie; activité antioxydante.

Keywords : ethnomedicinal, Crateva adansonii; Phytochemistry; Chromatography; antioxidant activity.

Publication date 31/10/2020, http://m.elewa.org/Journals/about-japs/

1 RÉSUMÉ

Crateva adansonii constitue l'une des espèces prisées au sein de la population béninoise pour ses nombreuses vertus thérapeutiques et nutritionnelles. La présente étude intitulée a été initiée pour garantir et promouvoir une meilleure utilisation de Crateva adansonii dans le traitement des maladies. L'étude ethnomédicinale a été réalisée au moyen des interviews semi structuré auprès des herboristes et des consommateurs de Cotonou et de Dassa. Le screening phytochimique a été effectué par chromatographie sur couche mince (CCM) doublé du dosage spectrophotométrique des métabolites secondaires. Les méthodes FRAP et DPPH ont servi à la détermination du pouvoir antioxydant et la CI 50\% a été calculée. Les enquêtés ethnomédicinales ont montré que Crateva adansonii s'utilise au Bénin aussi bien à des fins thérapeutiques, alimentaire que médico-magique. La forme galénique de ses organes les plus utilisées (tiges feuillées) est la décoction par voie orale. La phytochimie a révélé, en plus des principaux groupes phytochimiques (composés phénoliques, composés azotés, composés stéroïdes et terpénoïdes et mucilages), la présence des huiles essentielles, des lignanes et des pigments anthocyaniques. Dans la gamme de concentrations utilisées $\left(0,23-30 \mu \mathrm{g} \cdot \mathrm{ml}^{-1}\right)$, le pouvoir antioxydant de l'extrait varie de $5-58 \%$ (méthode $\mathrm{DPPH}$ ) et de 23,01 à 34,06mMolEAA/g (méthode FRAP). Quant au dosage des métabolites secondaires, l'extrait éthanolique de Crateva adansonii renferme respectivement 12,85 mg Eq Cat/100 mg de tannins totaux, 29,64 mg Eq Quer/100 $\mathrm{mg}$ de flavonoïdes totaux et 3,51 $\mathrm{mg}$ Eq ac. Galic/100 mg de phénoliques totaux. 


\section{ABSTRACT}

Crateva adansonii is one of the species prized by the Beninese population for its many therapeutic and nutritional benefits. This titled study was initiated to ensure and promote better use of Crateva adansonii in the treatment of diseases. The ethnomedicinal study was carried out through semi-structured interviews with herbalists and consumers in Cotonou and Dassa. The phytochemical screening was carried out by thin layer chromatography (TLC) coupled with the spectrophotometric assay of the secondary metabolites. The FRAP and DPPH methods were used to determine the antioxidant power and the IC $50 \%$ was calculated. Ethnomedicinal surveys have shown that Crateva adansonii is used in Benin for therapeutic, nutritional and medico-magical purposes. The dosage form of its most widely used organs (leafy stems) is the oral decoction. Phytochemistry revealed, in addition to the main phytochemical groups (phenolic compounds, nitrogen compounds, steroid and terpenoids compounds and mucilages), the presence of essential oils, lignans and anthocyanin pigments. In the range of concentrations used $(0.23$ - $30 \mu \mathrm{g} . \mathrm{ml}-1)$, the antioxidant power of the extract varies from 5 - 58\% (DPPH method) and from 23.01 to 34.06 mMolEAA / g (FRAP method). As for the determination of secondary metabolites, the ethanolic extract of Crateva adansonii contains respectively $12.85 \mathrm{mg}$ Eq Cat / $100 \mathrm{mg}$ of total tannins, $29.64 \mathrm{mg}$ Eq Quer / $100 \mathrm{mg}$ of total flavonoids and $3.51 \mathrm{mg} \mathrm{Eq}$ ac. Galic / $100 \mathrm{mg}$ total phenolics.

\section{INTRODUCTION}

Dans l'histoire du monde, les plantes sont toujours restées traditionnellement comme des remèdes pour prévenir ou guérir diverses maladies (Ramdane, 2018) car elles renferment plusieurs milliers de molécules dotées de potentiel thérapeutique énorme (Danton, 2017). D’après la littérature, $80 \%$ de la population mondiale contre $70 \%$ de la population béninoise majoritairement analphabète font recours à la Médecine Traditionnelle pour répondre à leurs besoins en santé (WHO/MS-Bénin, 2013). Le Bénin, de par sa position biogéographique offre une très grande diversité floristique. Au nombre des plantes médicinales (PM) les plus utilisées, Crateva adansonii figure en bonne position. Cette plante est utilisée au Bénin pour le traitement de

\section{MATÉRIEL ET MÉTHODES}

3.1 Étude ethnomédecinale

3.1.1 Cadre d'étude : Deux zones ont servi de cadre pour cette étude. Il s'agit de la l'hypertension artérielle (Tokoudagba et al., 2009 ; Olou et al., 2018), et le diabète chez les femmes enceintes à Cotonou (Agbankpé et al, 2014). Les tradithérapeutes utilisent Crateva adansonii au Togo, pour le traitement d'abcès, de plaies, d'infections bactériennes et de rhumatismes. Susceptible de posséder des propriétés antioxydantes et du fait des multiples vertus de réparation cellulaire que possède cette plante, ces feuilles sont très utilisées dans la pharmacopée africaine et indienne (Ahama et al, 2010). Au regard de ces nombreux usages dont fait l'objet Crateva adansonii, la présente étude est initiée pour contribuer à une utilisation saine de cette plante et promouvoir ainsi à la bonne santé de la population.

Commune de Cotonou et celle de DassaZoumè. 


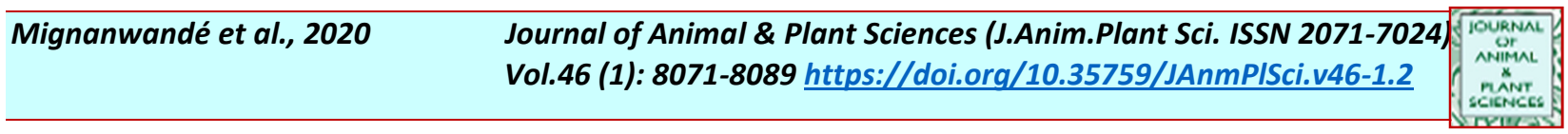

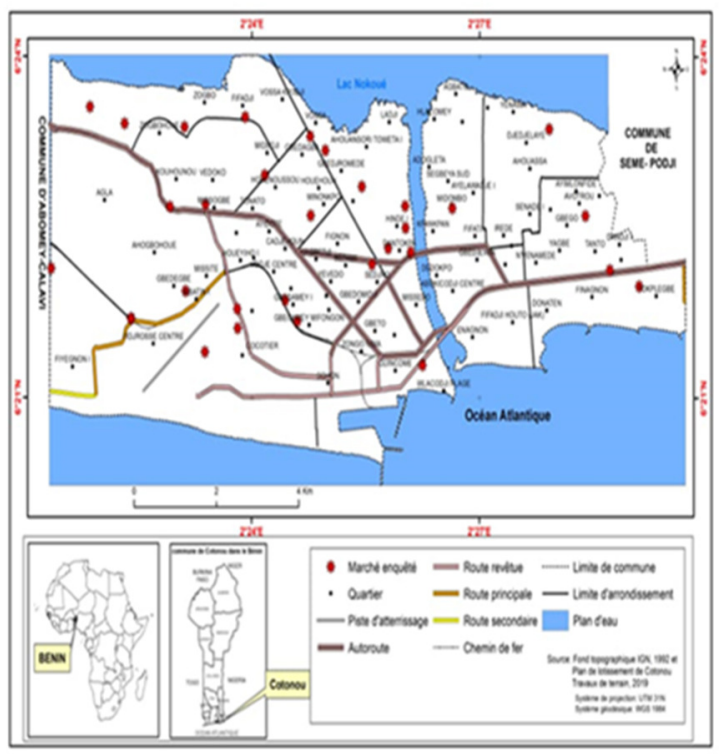

Figure 1 : Carte de la ville de Cotonou avec les différents marchés

3.1.2 Matériel : Le matériel utilisé pour l'étude ethnomédecinale est constitué composé de outils couramment utilisées pour les enquêtes de terrain à savoir : fiches d'enquête pour la collecte des données de terrain ; appareil photo numérique pour la prise des images de terrain ; enregistreur pour les interviews ; GPS pour la prise des coordonnées géographiques.

\subsubsection{Méthode de l'étude}

3.1.3.1 Type d'étude : Il s'est agi ici d'une étude descriptive analytique qui repose sur une enquête CAP (Connaissances ; Attitudes ; Pratiques) des populations sur $C$. adansonii.

3.1.3.2 Technique de l'étude: L'interview semi structuré (N'guessan et al., 2009) a été utilisé pour les enquêtés.

3.1.3.3 Technique d'échantillonnage: Les phytothérapeutes ont été choisis en tenant compte de trois critères

être une personne adulte et disposé d'une décennie d'expérience dans le domaine de vente de plantes médicinales ;

pouvoir s'exprimer dans l'une des langues locales retenues (Fon, Goun, Mina, Idacha);

accepter consacrer du temps pour l'interview.

3.1.3.4 Les échantillons : Les échantillons considérés sont de deux ordres : les herboristes

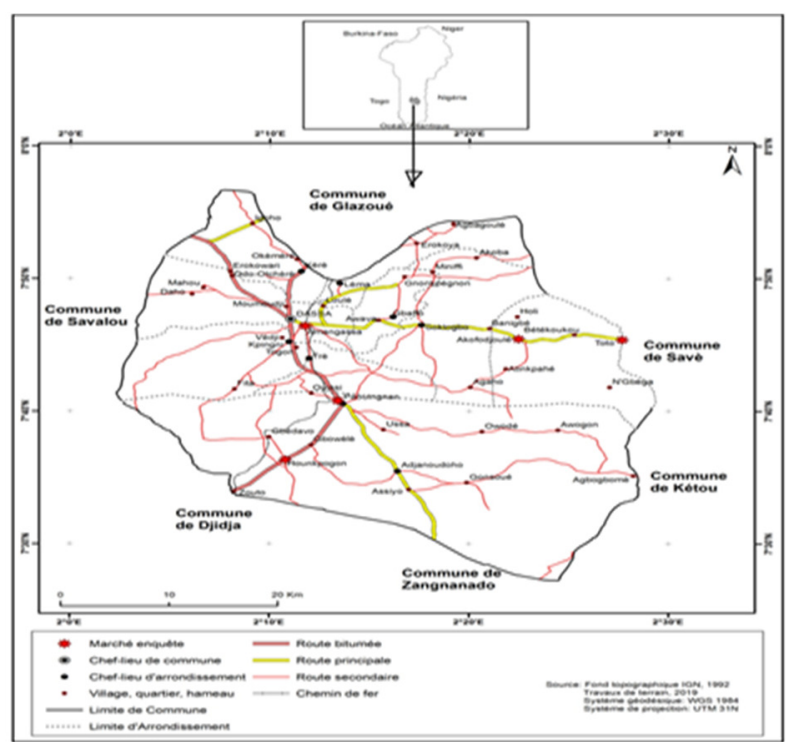

Figure 2 : Carte de la ville de Dassa-Zoumè avec les différents marchés

au niveau des marchés et les consommateurs au sein des ménages.

Herboristes: Trente-quatre (34) marchés de Cotonou et trois (03) marchés de Dassa ont été retenus pour mener les enquêtes ethnomédicinales. Il s'est agi d'une méthode de choix raisonné ne tenant pas compte de la catégorie des marchés et de l'importance du nombre d'herboristes présents dans ces marchés. Cette méthode vise une large représentativité l'échantillon et une fiabilité des résultats. Au niveau des marchés il a s'est agi d'un échantillonnage systématique. Entrer dans le marché par la grande rue, le premier herboriste a été choisi, s'il répond aux critères d'inclusion sus-cités, sinon on passe au suivant avant d'effectuer un bond de 4 dans le marché de Dantokpa et un bond de 2 dans les marchés secondaires.

- Les personnes enquêtées :A Cotonou, 115 personnes ont été interrogées dont 76 herboristes et 39 consommateurs. Dans la commune de Dassa-Zoumè 57 personnes ont été prises en compte à raison de 23 herboristes et 34 consommateurs. Au total 172 personnes ont été enquêtées. 
3.1.3.5 Analyse statistique des données : Les données recueillies à la suite des enquêtes ont été traitées en utilisant le logiciel tableur Excel 2010 et les analyses statistiques ont été faites grâce à IBM SPSS statistics 19.

3.1.3.6 Aspects éthique et déontologique : A ce niveau, il a été question de respecter essentiellement les règles d'éthique et de déontologie en matière de recherche scientifique. Dans ce cadre, la plante a été certifiée à l'Herbier National du Bénin sous le numéro YH 269 / HNB. Les règles saines pour la collecte et le traitement des données (enquêtes et interviews) ont été observées.

\subsection{Etude phytochimique}

3.2.1 Matériel: Le matériel des recherches phytochimiques a été regroupé en trois catégories à savoir le matériel végétal, le matériel technique et les réactifs.

\subsubsection{Méthodes}

3.2.2.1 Extraction du matériel végétal : L'extrait éthanolique a été obtenu par macération (Lehout et Maya, 2015 ; Hind, 2017). Ainsi, $100 \mathrm{~g}$ de la poudre de Crateva adansonii ont été soumises à une agitation mécanique pendant 24 heures avec $500 \mathrm{ml}$ d'éthanol. Le mélange obtenu après macération a été filtré respectivement sur tissu, coton fibre et papier Wattman. Chaque niveau de filtration est répété trois (03) fois. Le mac est ensuite soniqué puis filtré après ajout de $500 \mathrm{ml}$ d'éthanol. Le filtrat d'éthanol recueilli a été concentré sous pression à l'aide d'un rotavapor (BUCHI Rotavapor RII). L'extrait humide est recueilli dans des boîtes de pétri placé à l'étuve pour séchage puis raclé dans des piluliers en verre stérile et conservé à $4^{\circ} \mathrm{C}$ pour les différents tests.

\subsubsection{Screening phytochimique}

Criblage standard: Le criblage standard est réalisé selon la méthode de Houghton et Raman (1998) revue et adaptée aux conditions du Laboratoire de Pharmacognosie des Huiles Essentiels (LaPHE). Les tests de caractérisation sont basés sur des réactions de coloration précipitation différentielles Houghton et Raman (1998).
Chromatographie sur Couche Mince: Les tests sur plaque chromatographique (Bladt et Wagner, 2001) basé sur la détection des différentes familles de métabolites secondaires par la chromatographie sur couche mince (CCM) ont été utilisés. Dans cette méthode, la séparation des métabolites d'un extrait se fait suivant deux phases, l'une mobiles et l'autre stationnaire (plaque de silice). $10 \mu \mathrm{l}$ de chaque extraits préparé à $5 \mathrm{mg} / \mathrm{ml}$ sur plaques $\mathrm{CCM}$ (silica gel $60 \mathrm{~F}_{254}$, support rigide en aluminium, Merck). Les constituants séparés sont ensuite révélés a l'UV $254 \mathrm{~nm}$ et $365 \mathrm{~nm}$ et/ou par un réactif chimique approprié. Les grands groupes de métabolites secondaires recherchés sont les coumarines, les flavonoïdes, les tannins, les triterpènes, les alcaloïdes, les dérivées anthracéniques, les saponines, les naphtoquinones, les pigments.

\subsubsection{Quantification des métabolites} secondaires

Dosage des composés phénoliques totaux : La teneur en composés phénoliques de l'extrait éthanolique a été estimée par la méthode de Folin-Ciocalteu (Li et al., 2007 ; Mnayer, 2014). Basée sur la réduction en milieux alcalin de la mixture phosphotungstique $\left(\mathrm{WO}_{4}{ }^{2-}\right)$ phosphomolybdique $\left(\mathrm{MoO}_{4}{ }^{2-}\right)$ de réactif de Folin par les groupements oxydables des composés phénoliques, cette méthode conduit à la formation de produits de réduction de couleur bleue. Ces derniers présentent un maximum d'absorption à $765 \mathrm{~nm}$ dont l'intensité est proportionnelle à la quantité de polyphénols présents dans l'échantillon (Amoussa et al., 2015; Sharmin et al., 2018). Les extraits sont dissous dans du méthanol pour avoir une concentration de $1 \mathrm{mg} / \mathrm{ml}$, puis dilués au 1/100 dans l'eau distillée. Ensuite, $1 \mathrm{ml}$ de réactif de Folin 2N (10 fois dilué) est ajouté à $200 \mu \mathrm{l}$ de la solution d'extrait dilué. Après cinq minutes, $800 \mu 1$ d'une solution de carbonate de sodium (1M) sont additionnés au milieu réactionnel qui est à nouveau incubé pendant 02 heures à la température ambiante. A l'aide d'un spectrophotomètre (UV-1600 PC), l'absorbance est mesurée à $765 \mathrm{~nm}$. Les teneurs en 
phénoliques totaux sont déterminées à l'aide d'une courbe de calibration ou d'étalonnage linéaire $\left(y=0,043 x-0,051 ; R^{2}=0,994\right)$ tracée en utilisant des concentrations précises d'acide gallique $\left(0-60 \mathrm{mg} / \mathrm{l} ; \mathrm{R}^{2}=0,994\right)$ comme substance de référence. Les résultats sont calculés suivant l'équation 1 : $\left(C=\frac{\mathrm{cxD}}{\mathrm{Ci}} \times 100\right)$ et exprimés en mgEAG/100mg qui se lit milligramme équivalent acide gallique par $100 \mathrm{mg}$ d'extrait sec (Sharmin et al., 2018).

$\mathrm{C}=$ Concentration en phénoliques totaux en $\mathrm{mg}$ EAG/100 mg d'extrait sec $\mathrm{c}=$ Concentration de l'échantillon lue

$\mathrm{D}=$ Facteur de dilution

$\mathrm{Ci}=$ Concentration de la solution initiale

Dosage des flavonoïdes totaux : Les flavonoïdes totaux ont été quantifiés par la méthode du trichlorure d'aluminium (Nadhiya et Vijayalakshmi, 2014). Cette Méthode évalue l'ensemble des composés réagissant avec le trichlorure d'aluminium $\left(\mathrm{AlCl}_{3}\right)$. Les extraits sont préparés à $0,1 \mathrm{mg} / \mathrm{ml}$ dans du méthanol. Ensuite, $1 \mathrm{ml}$ d'échantillon est ajouté à $1 \mathrm{ml}$ de la solution d'AlCl $\mathrm{Al}_{3}$ (2\% dans le méthanol). L'absorbance est lue à $430 \mathrm{~nm}$, après 10 minutes de réaction. Un mélange de $1 \mathrm{ml}$ d'extrait et de $1 \mathrm{ml}$ du méthanol sans $\mathrm{AlCl}_{3}$ a servi de blanc. Trois analyses (triplet) ont été effectuées pour chaque concentration de l'extrait. Le résultat donné est la moyenne des trois lectures. La concentration des flavonoïdes est déduite à partir d'une courbe d'étalonnage $\left(\mathrm{y}=0,325 \mathrm{x}-0,363 ; \mathrm{R}^{2}=0,995\right)$ établie avec la quercétine $(0-100 \mathrm{mg} / \mathrm{l})$. Elle est exprimée en milligramme équivalent de quercétine par 100 milligramme d'extrait sec (mg EQ/100 mg) suivant l'équation 1: ( $\mathrm{C}=$ $\left.\frac{\mathrm{cxD}}{\mathrm{Ci}} \times 100\right)$.

$\mathrm{C}=$ Concentration en flavonoïdes totaux en $\mathrm{mg}$

EQ/100 mg d'extrait sec

$\mathrm{c}=$ Concentration de l'échantillon lue

$\mathrm{D}=$ Facteur de dilution

$\mathrm{Ci}=$ Concentration de la solution initiale

Dosage des tanins condensés: Les tanins sont des substances naturelles polyphénoliques, hydrosolubles à saveur astringente, ayant en commun la propriété de tanner la peau. Les tanins condensés sont déterminés ici par la méthode à la vanilline en milieu acide (Benchegra et Haddad, 2013 ; Mahmoudi et al., 2013) revue et adaptée aux conditions du LBSNB. Cette méthode est basée sur la capacité de la vanilline à réagir avec les unités des tanins condensés en présence d'acide pour produire un complexe coloré. La réactivité de la vanilline avec les tanins n'implique que la première unité du polymère. La solution mère d'extrait de Crateva adansonii est préparée à une concentration de $1 \mathrm{mg} / \mathrm{ml}$ dans du méthanol. Ensuite, prélever $400 \mu \mathrm{l}$ de cette solution mère et y ajouter $3 \mathrm{ml}$ de vanilline à $4 \%$. Additionner à ce mélange $1500 \mu \mathrm{l}$ d'acide chlorhydrique ( $\mathrm{HCl})$. Le blanc est préparé en remplaçant le réactif par le mélange méthanol-acide. Les tubes sont maintenus à $30^{\circ} \mathrm{C}$ pendant vingt minutes, dans une chambre noire. L'absorbance est lue à 500nm. Les teneurs en tanins condensés sont déterminées à l'aide d'une courbe de calibration ou d'étalonnage linéaire $\left(y=0,043 x-0,051 ; R^{2}=0,994\right)$ tracée en utilisant des concentrations précises d'acide gallique $\left(0-60 \mathrm{mg} / 1, \mathrm{R}^{2}=0,994\right)$ ou catéchine comme substance de référence. Les résultats sont calculés suivant l'équation $\left(\mathbf{C}=\frac{\boldsymbol{c x D}}{\boldsymbol{C} \boldsymbol{i}} \boldsymbol{x} \mathbf{1 0 0}\right)$ et exprimés en milligramme équivalent d'acide gallique par 100 milligrammes d'extrait sec (mgEAG/100mg).

$\mathrm{C}=$ Concentration en tanins condensés en $\mathrm{mg}$ EAG/100 mg d'extrait sec

$\mathrm{c}=$ Concentration de l'échantillon lue

$\mathrm{D}=$ Facteur de dilution

$\mathrm{Ci}=$ Concentration de la solution initiale

\subsubsection{Méthode FRAP}

La méthode FRAP (Ferric Reducing Antioxidant Power) est basée sur l'habilité des extraits à réduire l'ion ferrique $\left(\mathrm{Fe}^{3+}\right)$ présent dans le complexe ferricyanure de potassium $\left[\mathrm{K}_{3} \mathrm{Fe}(\mathrm{CN})\right.$ 6] en ion ferreux $\left(\mathrm{Fe}^{2+}\right)$ par un antioxydant. $\mathrm{La}$ réaction est révélée par le virage de la couleur jaune du fer ferrique $\left(\mathrm{Fe}^{3+}\right)$ à la couleur bleu-vert du fer ferreux $\left(\mathrm{Fe}^{2+}\right)$. L'intensité de cette coloration correspondant au pouvoir antioxydant de chaque extrait a été évalué par la méthode spectrophotométrique à 700nm (Saeed 
et al., 2012 ; Mnayer, 2014). Un volume de $2 \mathrm{ml}$ de chaque échantillon $(100 \mu \mathrm{g} / \mathrm{ml})$ a été mélangé avec $2 \mathrm{ml}$ de tampon phosphate $(0,2 \mathrm{M}, \mathrm{pH} 6,6)$ et $2 \mathrm{ml}$ de la solution aqueuse $(1 \%)$ d'hexacyanoferrate de potassium $\left[\mathrm{K}_{3} \mathrm{Fe}(\mathrm{CN})_{6}\right]$. Le mélange a été incubé à $50^{\circ} \mathrm{C}$ pendant $20 \mathrm{~min}$ suivi par l'addition de $2 \mathrm{ml}$ d'acide trichloroacétique $(10 \%)$. Le mélange a été centrifugé à $3000 \mathrm{tr} / \mathrm{min}$ pendant 10 minutes afin de recueillir la couche supérieure de la solution. Un volume de $2 \mathrm{ml}$ du surnageant ont été ensuite mélangés au même volume d'eau distillée et $0,4 \mathrm{ml}$ d'une solution aqueuse fraîchement préparée de $\mathrm{FeCl}_{3}(0,1 \%)$ a été ajoutée. Après 10 minutes de réaction, l'absorbance a été lue à $700 \mathrm{~nm}$. Le pouvoir réducteur est déduit à partir d'une courbe d'étalonnage ( $\left.\mathrm{y}=0,028 \mathrm{x}-0,024 ; \mathrm{R}^{2}=0,995\right)$ établie avec l'acide ascorbique $(0-100 \mathrm{mg} / \mathrm{l})$. Le pouvoir réducteur est exprimé en $\mu \mathrm{mol}$ Equivalent Acide Ascorbique par gramme d'extrait sec ( $\mu$ molEAA/g). L'acide ascorbique a été utilisé comme référence.

\subsubsection{Méthode DPPH : L'activité} antiradicalaire des extraits traduit leur aptitude à piéger les radicaux libres de l'organisme.

\section{RÉSULTATS}

\subsection{Etude ethnomédicinale}

4.1.1 Profil des personnes enquêtées à Cotonou et à Dassa-Zoumè : L'enquête ethnomédicinale à Cotonou a révélé un pourcentage de femmes enquêtées $(79,13 \%)$ supérieur aux hommes (20,87\%). Les âges évoluent de 30 à 90 ans et la classe modale est de 45 à 60 ans (60,0\%). L'ethnie dominante est le fon. Plus de la moitié des personnes enquêtées n'ont aucun niveau scolaire $(71,30 \%)$. La religion catholique est dominante. A Dassa-Zoumè la
L'activité antiradicalaire de l'extrait éthanolique a été évaluée par la méthode spectrophotométrique au 2,2 diphényl-1picrylhydrazyl (Mnayer, 2014; Lagnika et al., 2016). Le principe repose sur la réduction du radical DPPH qui passe de la couleur violette $(\mathrm{DPPH} \bullet)$ à la couleur jaune (DPPH-H). Cette capacité de réduction est déterminée par une diminution de l'absorbance induite par les substances antiradicalaires (Majhenic et al., 2007 ; Talbi et al., 2015). Une gamme de concentrations $(0,0078$ à $1 \mathrm{mg} / \mathrm{ml})$ obtenue à partir d'une solution mère d'extrait à $1 \mathrm{mg} / \mathrm{ml}$ dans du méthanol a permis d'évaluer l'activité antiradicalaire des extraits. Le milieu réactionnel est composé de $0,75 \mathrm{ml}$ d'extrait et $1,5 \mathrm{ml}$ d'une solution méthanolique de DPPH à $0,04 \mathrm{mg} / \mathrm{ml}$. Un témoin ne contenant pas d'extrait végétal (blanc d'échantillon) est préparé avec du méthanol. Les tubes ont été incubés à l'obscurité pendant $30 \mathrm{mn}$ à température ambiante et les densités optiques mesurées à $517 \mathrm{~nm}$ ont servi à calculer le pourcentage d'inhibition du radical DPPH proportionnel au pouvoir antiradicalaire de l'échantillon (Parejo et al., 2003 ; Mahmani, 2017).

majorité des personnes enquêtées sont des femmes $(85,96 \%)$. Les âges évoluent de 15 à 60 ans et la classe modale est de 30 à 45 ans $(70,18$ $\%$ ). Les deux ethnies prédominantes sont Mahi et Idaasha $(42,11 \%$ et $33,33 \%)$. La religion prédominante est le catholicisme (64,91\%). La majorité des enquêtés ne possède aucun niveau de scolarisation $(84,21 \%)$.

\subsubsection{Connaissances empiriques des enquêtés sur Crateva adansonii}


Tableau 1 : Connaissances empiriques Herboristes sur Crateva adansonii à Cotonou et Dassa

\begin{tabular}{|c|c|c|c|c|c|}
\hline \multirow{2}{*}{ Variables } & \multirow{2}{*}{ Modalités } & \multicolumn{2}{|c|}{ Herboristes Cotonou } & \multicolumn{2}{|c|}{ Herboriste Dassa } \\
\hline & & Effectifs & P. $(\%)$ & Effectifs & P. $(\%)$ \\
\hline $\begin{array}{l}\text { Types } \\
\text { d'usages } \\
\text { Total }\end{array}$ & $\begin{array}{l}\text { Thérapeutique } \\
\text { Alimentaire } \\
\text { Médico-magique } \\
\text { Ornemental }\end{array}$ & $\begin{array}{c}74 \\
0 \\
2 \\
0 \\
76\end{array}$ & $\begin{array}{c}97,37 \\
0,00 \\
2,63 \\
0,00 \\
\mathbf{1 0 0 , 0 0}\end{array}$ & $\begin{array}{c}21 \\
2 \\
0 \\
0 \\
23\end{array}$ & $\begin{array}{c}91,30 \\
8,70 \\
0,00 \\
0,00 \\
\mathbf{1 0 0 , 0 0}\end{array}$ \\
\hline $\begin{array}{l}\text { Types d'affections traitées } \\
\text { Total }\end{array}$ & $\begin{array}{l}\text { Affections digestives } \\
\text { Affections cutanées / dermatologique } \\
\text { Autres }\end{array}$ & $\begin{array}{c}41 \\
29 \\
6 \\
76\end{array}$ & $\begin{array}{c}53,95 \\
38,16 \\
7,89 \\
\mathbf{1 0 0 , 0 0}\end{array}$ & $\begin{array}{c}6 \\
13 \\
4 \\
23\end{array}$ & $\begin{array}{c}26,09 \\
56,52 \\
17,39 \\
\mathbf{1 0 0 , 0 0}\end{array}$ \\
\hline $\begin{array}{l}\text { Pathologies traitées } \\
\text { Total }\end{array}$ & $\begin{array}{l}\text { Hypertension } \\
\text { Abcès / plaies / infections / ulcères } \\
\text { Paludisme } \\
\text { Autres }\end{array}$ & $\begin{array}{c}7 \\
44 \\
20 \\
5 \\
76\end{array}$ & $\begin{array}{c}9,21 \\
57,89 \\
26,32 \\
6,58 \\
\mathbf{1 0 0 , 0 0}\end{array}$ & $\begin{array}{c}2 \\
6 \\
10 \\
5 \\
23\end{array}$ & $\begin{array}{c}8,70 \\
26,09 \\
43,48 \\
21,74 \\
\mathbf{1 0 0 , 0 0}\end{array}$ \\
\hline Organes végétaux utilisés & $\begin{array}{l}\text { Feuilles } \\
\text { feuilles et tige } \\
\text { Feuilles, tige et racine }\end{array}$ & $\begin{array}{c}12 \\
59 \\
5\end{array}$ & $\begin{array}{l}15,79 \\
77,63 \\
6,58\end{array}$ & $\begin{array}{c}4 \\
17 \\
2\end{array}$ & $\begin{array}{c}17,39 \\
73,91 \\
8,70\end{array}$ \\
\hline $\begin{array}{l}\text { Total } \\
\text { Mode de préparation }\end{array}$ & $\begin{array}{l}\text { Décoction } \\
\text { ramollissement / macération } \\
\text { Infusion } \\
\text { Autres }\end{array}$ & $\begin{array}{c}76 \\
72 \\
0 \\
2 \\
2\end{array}$ & $\begin{array}{c}\mathbf{1 0 0 , 0 0} \\
94,74 \\
0,00 \\
2,63 \\
2,63\end{array}$ & $\begin{array}{c}23 \\
20 \\
1 \\
1 \\
1\end{array}$ & $\begin{array}{c}\mathbf{1 0 0 , 0 0} \\
86,95 \\
4,35 \\
4,35 \\
4,35\end{array}$ \\
\hline $\begin{array}{l}\text { Total } \\
\text { Voie d'administration }\end{array}$ & $\begin{array}{l}\text { Orale } \\
\text { Cutanée } \\
\text { Anale/oculaire } \\
\text { Autre }\end{array}$ & $\begin{array}{c}76 \\
70 \\
6 \\
0 \\
0\end{array}$ & $\begin{array}{c}\mathbf{1 0 0 , 0 0} \\
92,11 \\
7,89 \\
0,00 \\
0,00\end{array}$ & $\begin{array}{c}23 \\
17 \\
5 \\
0 \\
1\end{array}$ & $\begin{array}{c}\mathbf{1 0 0 , 0 0} \\
73,91 \\
21,74 \\
0,00 \\
4,35\end{array}$ \\
\hline $\begin{array}{l}\text { Total } \\
\text { Risques connues } \\
\text { Total }\end{array}$ & $\begin{array}{l}\text { Aucun } \\
\text { Anémie } \\
\text { Autre }\end{array}$ & $\begin{array}{c}76 \\
40 \\
36 \\
0 \\
76\end{array}$ & $\begin{array}{c}\mathbf{1 0 0 , 0 0} \\
52,63 \\
47,37 \\
0,00 \\
\mathbf{1 0 0 , 0 0}\end{array}$ & $\begin{array}{c}23 \\
15 \\
8 \\
0 \\
23\end{array}$ & $\begin{array}{c}\mathbf{1 0 0 , 0 0} \\
65,22 \\
34,78 \\
0,00 \\
\mathbf{1 0 0 , 0 0}\end{array}$ \\
\hline
\end{tabular}


Tableau 2 : Connaissances empiriques consommateurs sur Crateva adansonii à Cotonou et Dassa

\begin{tabular}{|c|c|c|c|c|c|}
\hline \multirow{2}{*}{ Paramètres } & \multirow{2}{*}{ Modalités } & \multicolumn{2}{|c|}{ Consommateur Cotonou } & \multicolumn{2}{|c|}{ Consommateurs Dassa } \\
\hline & & Effectifs & P. $(\%)$ & Effectifs & P. $(\%)$ \\
\hline \multirow{4}{*}{$\begin{array}{l}\text { Types } \\
\text { d'usages }\end{array}$} & Thérapeutique & 39 & 100,00 & 34 & 100,00 \\
\hline & Alimentaire & 0 & 0,00 & 0 & 0,00 \\
\hline & Médico-magique & 0 & 0,00 & 0 & 0,00 \\
\hline & Ornemental & 0 & 0,00 & 0 & 0,00 \\
\hline \multirow{2}{*}{ Total } & & 39 & 100,00 & 34 & 100,00 \\
\hline & Affections digestives & 17 & 43,59 & 4 & 11,76 \\
\hline \multirow[t]{2}{*}{ Types d'affections traitées } & Affections cutanées / dermatologique & 18 & 46,15 & 20 & 58,82 \\
\hline & Autres & 4 & 10,26 & 10 & 29,41 \\
\hline \multirow[t]{2}{*}{ Total } & & 39 & 100,00 & 34 & 100,00 \\
\hline & Hypertension & 4 & 10,26 & 2 & 5,88 \\
\hline \multirow{3}{*}{ Pathologies traitées } & Abcès / plaies / infections / ulcères & 16 & 41,03 & 12 & 35,29 \\
\hline & Paludisme & 12 & 30,77 & 15 & 44,12 \\
\hline & Autres & 7 & 17,95 & 5 & 14,71 \\
\hline \multirow[t]{2}{*}{ Total } & & 39 & 100,00 & 34 & 100,00 \\
\hline & Fenilles & 7 & 17,95 & 4 & 11,76 \\
\hline \multirow[t]{2}{*}{ Organes végétaux utilisés } & feuilles et tige & 30 & 76,92 & 26 & 76,47 \\
\hline & feuilles tige et racine & 2 & 5,13 & 4 & 11,76 \\
\hline Total & & 39 & 100,00 & 34 & 100,00 \\
\hline \multirow{4}{*}{ Mode de préparation } & Décoction & 26 & 66,67 & 28 & 82,35 \\
\hline & ramollissement / macération & 1 & 3900,00 & 3 & 10200,00 \\
\hline & Infusion & 9 & 23,08 & 3 & 8,82 \\
\hline & Autres & 3 & 7,69 & 0 & 0,00 \\
\hline \multirow[t]{2}{*}{ Total } & & 39 & 100,00 & 34 & 100,00 \\
\hline & Orale & 34 & 87,18 & 25 & 73,52 \\
\hline \multirow[t]{2}{*}{ Voie d'administration } & Cutanée & 5 & 12,82 & 9 & 26,47 \\
\hline & anale/oculaire & 0 & 0,00 & 0 & 0,00 \\
\hline Total & & 39 & 100,00 & 34 & 100,00 \\
\hline \multirow{2}{*}{ Risques connues } & Aucun & 19 & 48,72 & 15 & 44,12 \\
\hline & Anémie & 20 & 51,28 & 19 & 55,88 \\
\hline Total & & 39 & 100,00 & 34 & 100,00 \\
\hline
\end{tabular}


4.1.3 Connaissances des enquêtés sur la posologie de Crateva adansonii Connaissances des Herboristes sur la posologie de Crateva adansonii

Tableau 3: Connaissances des Herboristes sur la posologie de Crateva adansonii

\begin{tabular}{|c|c|c|c|c|}
\hline \multirow{2}{*}{ Variables / Modalités } & \multicolumn{2}{|c|}{ Herboristes Cotonou } & \multicolumn{2}{|c|}{ Herboristes Dassa-Zoumè } \\
\hline & Effectifs & P. $(\%)$ & Effectifs & P. $(\%)$ \\
\hline \multicolumn{5}{|c|}{ Définition du dosage } \\
\hline Non défini & 56 & 73,68 & 19 & 82,61 \\
\hline défini & 20 & 26,32 & 4 & 17,9 \\
\hline Total & 76 & 100,00 & 23 & 100,00 \\
\hline \multicolumn{5}{|c|}{ Nombre de prise par jour } \\
\hline un & 0 & 0,00 & 0 & 0,00 \\
\hline deux & 56 & 73,68 & 16 & 69,57 \\
\hline trois & 11 & 14,47 & 7 & 30,43 \\
\hline non défini & 9 & 11,84 & 0 & 0,00 \\
\hline Total & 76 & 100,00 & 23 & 100,00 \\
\hline \multicolumn{5}{|c|}{ Période de prise } \\
\hline Non défini & 5 & 6,58 & 0 & 0,00 \\
\hline Matin & 0 & 0,00 & 0 & 0,00 \\
\hline matin et soir & 60 & 78,95 & 16 & 69,57 \\
\hline matin, midi et soir & 11 & 14,47 & 7 & 30,43 \\
\hline Total & 76 & 100,00 & 23 & 100,00 \\
\hline \multicolumn{5}{|c|}{ Durée de traitement } \\
\hline $1 j r-1$ semaine & 41 & 53,95 & 10 & 43,48 \\
\hline deux semaines & 4 & 5,26 & 4 & 17,39 \\
\hline jusqu'à satisfaction & 30 & 39,47 & 9 & 39,13 \\
\hline non définit & 1 & 1,32 & 0 & 0,00 \\
\hline Total & 76 & 100,00 & 23 & 100,00 \\
\hline
\end{tabular}

Connaissances des consommateurs sur la posologie de Crateva adansonii

Tableau 4: Connaissances des consommateurs sur la posologie de Crateva adansonii

\begin{tabular}{|c|c|c|c|c|}
\hline \multirow{2}{*}{$\begin{array}{l}\text { Variables / } \\
\text { modalités }\end{array}$} & \multicolumn{2}{|c|}{ Consommateurs Cotonou } & \multicolumn{2}{|c|}{ Consommateurs Dassa } \\
\hline & Effectifs & $P .(\%)$ & Effectifs & $P .(\%)$ \\
\hline \multicolumn{5}{|c|}{ Définition du dosage } \\
\hline Non défini & 33 & 84,62 & 34 & 100,00 \\
\hline Défini & 6 & 15,38 & 0 & 0,00 \\
\hline Total & 39 & 100,00 & 34 & 100,00 \\
\hline \multicolumn{5}{|c|}{ Nombre de prise ou d'application } \\
\hline$U_{n}$ & 0 & 0.00 & 0 & 0,00 \\
\hline Deux & 21 & 53,85 & 18 & 52,94 \\
\hline Trois & 18 & 46,15 & 11 & 32,35 \\
\hline non défini & 0 & 0,00 & 5 & 14,71 \\
\hline Total & 39 & 100,00 & 34 & 100,00 \\
\hline \multicolumn{5}{|c|}{ Période de prise } \\
\hline Non défini & 0 & 0,00 & 4 & 11,76 \\
\hline Matin & 0 & 0,00 & 0 & 0,00 \\
\hline matin et soir & 21 & 53,85 & 19 & 55,88 \\
\hline
\end{tabular}


matin, midi et soir

Total

\section{Durée de traitement}

1 jr- 1 semaine

deux semaines

jusqu'à satisfaction

non définit

Total

\begin{tabular}{c|c}
18 & 46,15 \\
$\mathbf{3 9}$ & $\mathbf{1 0 0 , 0 0}$ \\
\hline $\boldsymbol{t}$ & \\
23 & 58,97 \\
5 & 12,82 \\
11 & 28,21 \\
0 & 0,00 \\
$\mathbf{3 9}$ & $\mathbf{1 0 0 , 0 0}$
\end{tabular}

32,35

100,00

29,41

14,71

55,88

0,00

100,00

4.2. Etude phytochimique

\subsubsection{Rendement de l'extraction}

Tableau 5 : rendement de l'extraction de Crateva adansonii

\begin{tabular}{l|l|l|l}
\hline Matériel végétal (g) & Solvant d'extraction & $\begin{array}{l}\text { Masse d'extrait } \\
\text { obtenu (g) }\end{array}$ & Rendement (\%) \\
\hline 2100 & $\mathrm{Et}-\mathrm{OH}$ & 176,264 & 8,39 \\
\hline
\end{tabular}

\subsubsection{Criblage standard}

Tableau 6 : Screening standard de Crateva adansonii

\begin{tabular}{|c|c|c|c|c|}
\hline \multicolumn{3}{|c|}{ Composés chimiques } & Observations & Résultats \\
\hline \multirow{2}{*}{$\begin{array}{l}\text { Tanins } \\
\text { Flavonö̈des }\end{array}$} & \multicolumn{2}{|c|}{$\begin{array}{l}\text { Tanins catéchiques } \\
\text { Tanins galliques }\end{array}$} & $\begin{array}{l}\text { Précipité Rose } \\
\text { Teinte Bleu }\end{array}$ & $\begin{array}{l}+++ \\
++\end{array}$ \\
\hline & \multicolumn{2}{|c|}{$\begin{array}{l}\text { Flavones } \\
\text { Leucoanthocyanes }\end{array}$} & $\begin{array}{l}\text { Coloration orangée } \\
\text { Coloration rouge cerise }\end{array}$ & $\begin{array}{l}++ \\
+++\end{array}$ \\
\hline \multicolumn{3}{|l|}{ Anthocyanes } & Coloration rouge & +++ \\
\hline $\begin{array}{l}\text { Dérivés } \\
\text { anthracéniques }\end{array}$ & $\begin{array}{l}\text { Libres } \\
\text { Combinés }\end{array}$ & $\begin{array}{l}\text { O-Hétérosides } \\
\text { C-Hétérosides } \\
\text { Composés } \\
\text { réducteurs }\end{array}$ & $\begin{array}{l}\text { Absence de Coloration rouge } \\
\text { Coloration rouge intense } \\
\text { Coloration rouge intense } \\
\text { Précipité rouge vif }\end{array}$ & $\begin{array}{l}- \\
+++ \\
+++ \\
++\end{array}$ \\
\hline \multicolumn{3}{|l|}{ Coumarines } & Absence de fluorescence & - \\
\hline \multicolumn{3}{|l|}{ Alcaloïdes } & Précipité Louche & ++ \\
\hline \multicolumn{3}{|l|}{ Stéroïdes } & $\begin{array}{l}\text { Absence de coloration rouge } \\
\text { pourpre }\end{array}$ & - \\
\hline \multicolumn{3}{|l|}{ Saponosides } & $\begin{array}{l}\text { Présence de mousse de } \\
\text { hauteur entre } 1 \mathrm{~cm} \text { et } 2 \mathrm{~cm}\end{array}$ & +++ \\
\hline \multicolumn{3}{|l|}{ Triterpènes } & Absence de coloration violette & - \\
\hline \multicolumn{3}{|l|}{ Cardenolides } & Absence de coloration bleue & - \\
\hline \multicolumn{3}{|l|}{ Mucilages } & $\begin{array}{l}\text { Présence } \\
\text { floconneux }\end{array}$ & + \\
\hline \multicolumn{3}{|c|}{ Dérivés cyanogéniques } & $\begin{array}{l}\text { Absence de coloration } \\
\text { marron }\end{array}$ & - \\
\hline \multicolumn{3}{|c|}{ Dérivés quinoniques } & $\begin{array}{l}\text { Absence de coloration rose ou } \\
\text { rouge violacée }\end{array}$ & - \\
\hline
\end{tabular}

« $+++»$ : Forte présence ; « $++»$ : Présence modérée ; «+» : Présence faible; «-» : absence 


\subsubsection{Chromatographie sur Couche Mince}

Tableau 7 : Chromatographie sur Couche Mince de Crateva adansonii.

\begin{tabular}{|c|c|c|c|c|c|}
\hline $\mathbf{N}^{\circ}$ & Familles & Réactifs & Colorations & Images & Résultats \\
\hline 1 & Alcaloïdes & Draggendorf & Marron, orange & & Présence \\
\hline 2 & Coumarines & NP/PEG & $\begin{array}{l}\text { Bleue intense, } \\
\text { bleue-vert, } \\
\text { Jaune, marron }\end{array}$ & & Présence \\
\hline 3 & $\begin{array}{l}\text { Dérivés } \\
\text { anthracéniques }\end{array}$ & KOH-ЕTOH 10\% & Rouge, jaune & & Présence \\
\hline 4 & Flavonoïdes & $\mathrm{NP} / \mathrm{PEG}$ & $\begin{array}{l}\text { Jaune orangé } \\
\text { Jaune verdâtre }\end{array}$ & & Présence \\
\hline 5 & $\begin{array}{l}\text { Huiles } \\
\text { essentielles }\end{array}$ & Vanillinesulfurique & Bleue, vert, rouge & & Présence \\
\hline 6 & Lignanes & Vanillinesulfurique & Bleu & & Absence \\
\hline
\end{tabular}




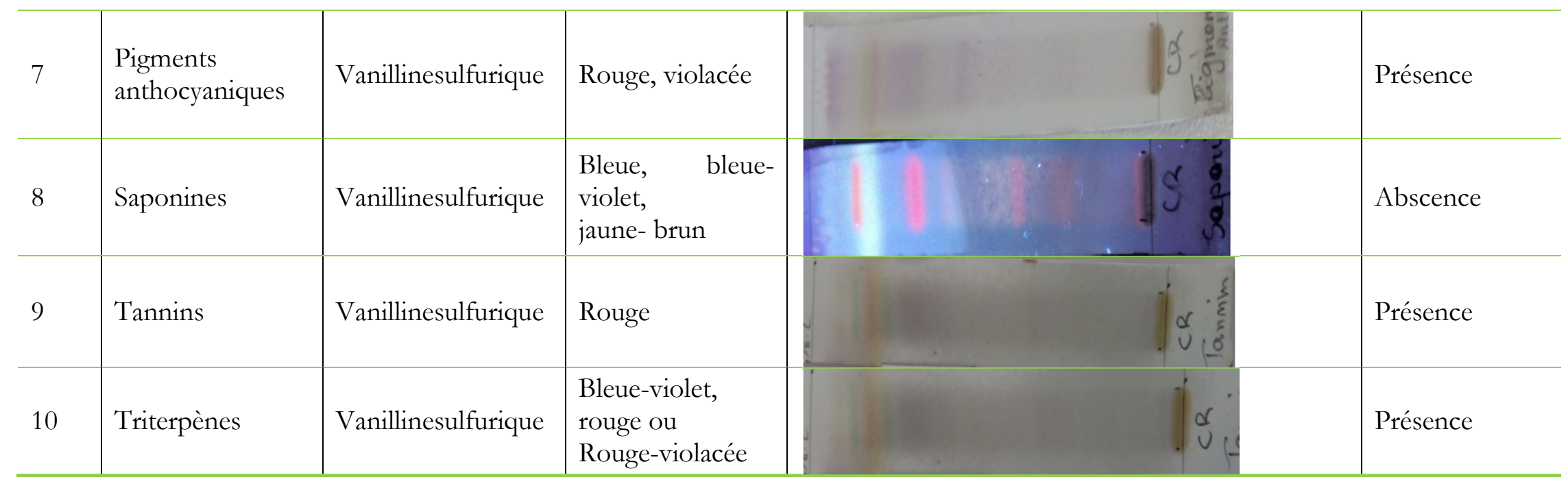




\subsubsection{Dosage des métabolites secondaires}

Tableau 8 : Dosage des métabolites secondaires

\begin{tabular}{l|l}
\hline Molécules recherchées & Concentration pour $\mathbf{1 0 0} \mathbf{m g}$ d'extract \\
\hline Phénoliques totaux & $3,51 \mathrm{mg} \mathrm{Eq}$ ac. Galic/100 mg \\
\hline Tanins totaux & $12,85 \mathrm{mg} \mathrm{Eq}$ Cat/100 mg \\
\hline Flavonö̈des totaux & $29,64 \mathrm{mg} \mathrm{Eq} \mathrm{Quer/100} \mathrm{mg}$ \\
\hline
\end{tabular}

4.2.5 Pouvoir antioxydant de Crateva adansonii par DPPH et CI 50\%

La figure 3 rend compte de l'activité anti radicalaire par la méthode DPPH.

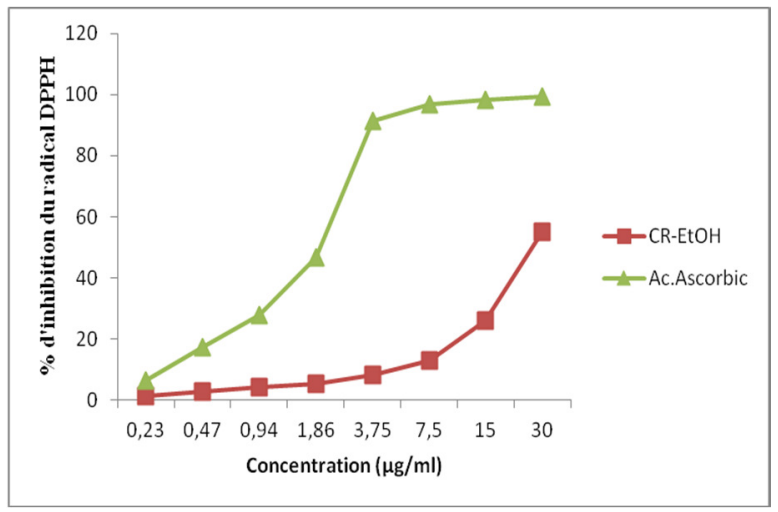

Figure 3: Capacité inhibitrice de l'extrait et de la molécule de référence

\subsubsection{Concentrations Inhibitrices à $50 \%$ de l'extrait et standard}

Tableau 9: Concentrations Inhibitrices à 50\% de l'extrait et standard

\begin{tabular}{l|l}
\hline Extrait / Fractions & Concentrations Inhibitrices $\mathbf{5 0} \% \mathbf{~ e n} \mathbf{~ m g} \cdot \mathbf{m l}^{\mathbf{1}}$ \\
\hline Crateva adansonii & 10,25 \\
\hline Acide ascorbique & 3,82 \\
\hline
\end{tabular}

\subsubsection{Pouvoir antioxydant par la méthode FRAP}

Tableau 10: Pouvoir antioxydant par la méthode FRAP

\begin{tabular}{|c|c|c|c|c|c|c|c|c|}
\hline Paramètres & \multicolumn{8}{|c|}{ Valeurs } \\
\hline $\begin{array}{l}\text { Concentration l'extrait } \\
\text { éthanolique }\left(\mu g . \mathrm{ml}^{1}\right)\end{array}$ & 0,23 & 0,47 & 0,94 & 1,86 & 3,75 & 7 & 15 & 30 \\
\hline Absorbance & 0,001 & 0,001 & 0,009 & 0,015 & 0,020 & 0,025 & 0,029 & 0,035 \\
\hline $\begin{array}{l}\text { Activité antioxydant } \\
\text { (mMol } E A A / g)\end{array}$ & 23,01 & 23,01 & 29,02 & 29,01 & 30,01 & 30,01 & 33,03 & 34,06 \\
\hline
\end{tabular}




\section{DISCUSSION}

La population de sexe féminin enquêtée domine largement celle de sexe masculin aussi bien à Cotonou qu'à Dassa-Zoumè. Ce fort taux des femmes enquêtées au détriment de celui des hommes est conforme aux résultats de certains auteurs (Mehdioui et Kahouadji, 2007 ; Agbankpé et al., 2014 ; Dougnon et al., 2016 ; Kpètèhoto et al., 2017). Ceci témoigne du rôle extrêmement prépondérant de la femme par coutume dans la chaîne de production et cuisson au sein des ménages particulièrement dans la filière de commercialisation des plantes médicinales. Nos résultats sont aussi en concordance avec ceux de Mehdioui et Kahouadji (2007) et Djègo et al. (2011) qui ont montré que les femmes constituent la grande masse détentrice du savoir phytothérapeutique traditionnel. Par contre, Dasylva (2001) à Dakar puis Gnagne et al. (2017) en Côte d'Ivoire ont montré que les hommes sont plus nombreux dans le commerce des plantes médicinales contrairement à nos résultats. Cette différence pourrait s'expliquer par les réalités socioculturelles entre les populations de Dakar, Bénin, Côte d'Ivoire et d'ailleurs. La majorité des vendeurs de PM, est analphabète. Ces résultats sont confirmés par les travaux de Fah et al., (2013), et de Dougnon et al., (2016), qui trouvent respectivement $92,86 \%$ et $60 \%$ d'analphabètes parmi les professionnels dans le domaine de vente de PM. L'enquête ethnomédicinale a révélé que Crateva adansonii est prioritairement utilisé à des fins thérapeutiques $(91,30$ - 100\%) mais également comme légumes feuilles $(8,70 \%)$. Ces résultats sont concordants avec ceux de Agbankpé et al., (2014), Olou et al., (2018) dont les conclusions ont montré que Crateva adansonii est une plante à la fois alimentaire, médicinale et médico-magique. Au nombre des diverses pathologies pour lesquelles Crateva adansonii est connu, les enquêtes ethnomédecinales ont montré qu'il est beaucoup plus utilisé dans le traitement de l'hypertension, des abcès, des plaies, des infections, des ulcères et du paludisme. Ces résultats confirment ceux de Olou et al., (2018), Agbankpé et al., (2014).
L'enquête ethnomédicinale menée auprès des populations de Cotonou et Dassa-Zoumè a révélé que les organes de $C$. adansonii les plus utilisés sont les tiges feuillées (76,47 - 77,63\%). La forme galénique dominante et la décoction $(66,67$ - 94,74\%) par voie orale $(73,52$ 92,11\%). D’après les enquêtés, l'abus de la consommation du jus de Crateva adansonii provoquerait l'anémie $(47,37$ - 55, 83\%) connu comme seul risque connu lié à l'utilisation. Ces résultats sont en concordance avec ceux de Dougnon et al., (2016) qui font observer que les feuilles sont les parties les plus usitées dont la décoction est la forme galénique utilisée et administrée par voie orale. Les données recueillies chez les enquêtés (herboristes et consommateurs) révèle qu'aucun dosage n'est défini $(73,68-82,61 \%)$ quant à l'utilisation de Crateva adansonii. Deux (69,57 - 78,95\%) périodes de prises ont été indiquées et sont généralement les matins et les soirs. La durée du traitement varie d'une semaine jusqu'à satisfaction complète $(53,95-55,88 \%)$. En guise de comparaison, ces résultats sont conformes à ceux de de Dougnon et al., (2016) qui ont montré qu'aucun dosage n'est défini par rapport à l'utilisation de Crateva adansonii et de certaines plantes. L'extrait éthanolique des tiges feuillées de Crateva adansonii a donné un rendement de $8.39 \%$. Dans une approche comparative, ce rendement est inférieur à ceux obtenus par Nounagnon et al., (2018) avec l'extrait aqueux $(16,7 \%)$ des feuilles de Crateva adansonii mais largement supérieur au rendement obtenu par ce dernier sur l'extrait hexanique $(0,66 \%)$ de la même plante. Les résultats du criblage standard révèlent la présence des trois groupes de constituants phytochimiques : les composés phénoliques, les composés azotés et les composés stéroïdes et terpénoïdes. Les composés phénoliques manifestent leur présence dans la plante à travers les tanins (catéchiques ; galliques), les flavonoïdes (flavones), les leuco-anthocyanes et les anthracéniques (libres) dans des proportions variables. Ces résultats sont confirmés par les 
travaux de Borokini et Omotayo (2012) ; Tsado et al., (2015), de Agbankpé et al., (2016) et Nounagnon, et al., (2018) qui ont abouti en partie au même résultat de screening phytochimique sur Crateva adansonii. La seule spécificité dans ces résultats est l'absence des leuco-anthocyanes. Cette différence pourrait s'expliquer par certains facteurs tels que le type de sol, la provenance des plantes et la période de récolte. Ce résultat est comparable à ceux de Yémoa 2008 et de Agbankpé et al., ; (2016) qui affirme que les tanins constituent le groupe chimique le plus fréquent. En plus des tanins, les alcaloïdes et les saponosides sont aussi des molécules très fréquentes (Borokini et Omotayo 2012 ; Agbankpé et al., 2016). Par contre les dérivés quinoniques et cyanogéniques, ne sont pas présents. Ces résultats sont confirmés par Nounagnon et al., (2018). La CCM de l'extrait éthanolique de Crateva adansonii a révélé la présence des alcaloïdes, des coumarines, des dérivées anthracéniques des flavonoides, des huiles essentielles, des lignanes, des pigments anthocyaniques, des tannins, des triterpènes et enfin l'absence des saponines. Ces résultats sont convergents avec ceux obtenus par (Diouf et al., 2015) sur l'extrait chloroformique qui a montré la présence des alcalö̈des, des tanins, des flavonoïdes, des polyphénols et l'absence des saponosides. En revanche, ces résultats de CCM sont divergents de ceux obtenus par (Diouf et al., 2015) avec les extraits méthanolique, cyclohexanique et aqueux. Des résultats de cette étude il peut être retenu que l'extraction éthanolique montre plus d'efficacité que celles cyclohexanique, aqueux et méthanolique car aucun de ces trois extraits n'a pu révéler à lui seul la présence des trois grands groupes phytochimiques. En continuité parfaite à l'établissement de la carte phytochimique de la plante, le dosage des molécules secondaires à savoir les tanins totaux, les flavonoïdes totaux et les phénoliques totaux dans l'extrait éthanolique a été réalisé. Ces résultats montrent que Crateva adansonii renferme plus de flavonoïdes totaux (29,64 mg Eq Quer/100 mg) que de tannins totaux $(12,85 \mathrm{mg}$ EqCat/100 $\mathrm{mg})$ et de polyphénols totaux $(3,51 \mathrm{mgEq}$ ac.Galic/100 $\mathrm{mg})$. En guise de comparaison, la gamme de concentrations en phénoliques totaux $(65,8$ $344,74 \mu \mathrm{g}$ Eq ac.Galic/100 mg) obtenue par Nounagnon et al., (2018) est largement inférieur à la valeur obtenue $(3510 \mathrm{mgEq}$ ac.Galic/100 mg) dans cette étude. Cette différence peut être due au site de récolte, de l'âge physiologique de la plante ou encore du moment de la récolte. Les résultats de l'activité antiradicalaire par la méthode DPPH de l'extrait éthanolique des tiges feuillées de Crateva adansonii ont révélé un pourcentage d'inhibition variant entre $5-58 \%$ dans la gamme de concentration allant de 0,23 $30 \mu \mathrm{g} / \mathrm{ml}$. Dans une étude réalisée par Ahama et al., 2010 sur Crateva adansonii de différents âges et récoltés à différents moments de la journée, le pouvoir antioxydant varie de 53,68-98,2\%. Ces résultats sont confirmés par ceux de Ahama et al., 2010 qui ont travaillé sur les feuilles jeunes et matures, de Crateva adansonii et ont obtenu un pouvoir antioxydant respectivement de 56,74\% et $63,34 \%$. Ces résultats montrent que les propriétés antioxydantes sont en étroite corrélation avec l'âge des feuilles qui diminuent des plus jeunes feuilles aux feuilles âgées (Ahama, et al., 2010). Il en résulte de la comparaison de ces résultats que la capacité antioxydante est dose dépendante. Dans une logique de confirmation des résultats du pouvoir antioxydant par la méthode FRAP, l'extrait éthanolique de Crateva adansonii a présenté un potentiel antiradicalaire moyen par la réduction du fer ferrique en fer ferreux compris entre 23,01 - 34,06mMol EAA/g. ces résultats s'assimilent à ceux de (Ahama, Quashie et al., 2010) qui ont abouti à la conclusion que les concentrations équivalentes en $\mathrm{Fe}^{2+}$ diminuent significativement lorsque le extrait provient des feuilles de plus en plus âgées. 


\section{CONCLUSION}

La flore béninoise dispose d'une richesse diversifiée de plantes médicinales utilisées par les populations pour la prévention et le traitement de différentes pathologies. Les résultats issus de cette enquête ethnobotanique ont révélé que Crateva adansonii est une plante bien connue et d'utilisation courante. Bien que de nombreuses régions détiennent des savoirs par rapport à son utilisation, les variantes observées dans cette dernière, au niveau des différentes communautés, ne sont pas significativement divergents. Nombreuses sont les pathologies

\section{RÉFÉRENCES}

Adomou AC., Yédomonhan H., Djossa B., Lègba SI., Oumorou M. et Akoègninou A. (2012). Etude Ethnobotanique des plantes médicinales vendues dans le marché d'Abomey-Calavi au Bénin. Int. J. Biol. Chem. Sci. 6 (2) : 745 - 772.

Agbankpe AJ., Dougnon TV., Bankole SH., Houngbegnon O., Dah-nouvlessounon D., Baba-moussa. (2016). In Vitro Antibacterial Effects of Crateva adansonii, $V$ ernonia amygdalina and Sesamum radiatum used for the Treatment of Infectious Diarrhoeas in Benin. Journal of Infectious Diseases \& Therapy.4:281. http://dx.doi.org/10.4172/23320877.1000281

Agbankpé AJ., Dougnon TV., Bankolé HS., Yèhouénou B., Yédomonhan H., Lègonou M., Dougnon TJ. (2014). Etude ethnobotanique des légumes feuilles thérapeutiques utilisés dans le traitement des diarrhées au sud-Bénin (Afrique de l'Ouest). Int. J. Biol. Chem. Sci. 8 (4) : $1784-1795$.

Agbankpé AJ., Bankolé SH., Assogba F., Dougnon TV., Yèhouénou B., Gbénou J., Baba-Moussa L. (2015). Phytochemical screening and cytotoxic analysis of three local vegetables used in the treatment of bacterial diarrhoea in southern Benin (West Africa) : A Comparative Study. BBJ., 9 (4) : 1 - 13. pour lesquelles la plante est utilisée. Des infections banales jusqu'aux plus complexes, des maladies ordinaires jusqu'à celles chroniques, Crateva adansonii manifeste une certaine efficacité. En somme, Crateva adansonii est une plante dont la composition qualitative et quantitative en métabolites secondaires dépend, comme pour beaucoup de plantes, des conditions environnementales. Crateva adansonii possède un pouvoir antioxydant qui probablement renforce le rôle des métabolites, surtout celui antibactérien, qu'il serait utile d'investiguer.

Ahama KYS., Quashie M-L A., Agbonon I., K Koumaglo K. (2010). Activités antioxydantes in vitro des feuilles de Crataeva adansonii. Revue Ivoirienne des Sciences et Technologie. Rev. Ivoir. Sci. Technol., 16 (2010) 153 - 164

Amoussa AMO. (2016). Phytochimie, activités antimicrobiennes et antioxydantes d'extraits et de molécules isolées des ecorces de Acacia ataxacantha DC. (Fabaceae). Thèse Unique de Doctorat à l'UAC. Ecole Doctorale Sciences de la Vie et de la Terre. FAST, 223 pages.

Attou A. (2011). Contribution à l'étude phytochimique et activités biologiques des extraits de la plante Ruta chalpensis (Fidjel) de la région d'Ain Témouchent. Mémoire du diplôme de Magister en biologie, option produits naturels : «Activités biologiques et synthèses ». Université Abou Bekr Belkaid Tlemcen (Algérie). 119 pages.

Benchegra H., Haddad D. (2013). Extraction et caractérisation des composés phénoliques de Myrtus communis L en vue de leur incorporation dans la margarine. Mémoire d'Ingénieur d'Etat en Contrôle de Qualité et Analyse. Université Abderrahmane MIRA de Bejaia. 78 pages

Borokini1 TI., and Omotayo FO. (2012). Phytochemical and ethnobotanical study 
of some selected medicinal plants from Nigeria Journal of Medicinal Plants Research Vol. 6(7), pp. 1106-1118, National Centre for Genetic Resources and Biotechnology, Moor Plantation, Ibadan, Oyo State, Nigeria. DOI: 10.5897/SRE09.525

Danton O. (2017). Extraction de substances naturelles antalgiques à partir de plantes utilisées dans la pharmacopée traditionnelle au Mali. Thèse de Doctorat, spécialité Chimie Organique. Université Clermont Auvergne. 255 pages

El Hadji Diouf G., Samb A., Sylla O., Kafia Ae., Diop M., Seck D., N'guessan K. (2015). Test phytochimique et insecticide de trois extraits organiques de feuilles de Ficus thonningii sur Callosobruchus maculatus Fabricius. Int. J. Biol. Chem. Sci. 8(6): 2588-2596. DOI

http:/ /dx.doi.org/10.4314/ijbcs.v8i6.20

Dougnon TV., Attakpa E., Bankolé H., Hounmanou YMG., Dèhou R., Agbankpè J., de Souza M., Fabiyi K., Gbaguidi F., Baba-Moussa L. (2016). Etude ethnobotanique des plantes médicinales utilisées contre une maladie cutanée contagieuse : La gale humaine au Sud-Bénin. Revue CAMES -Série Pharm. Méd. Trad. Afr., 18 (1) : 16 -22.

Fah L., Klotoé JR., Dougnon V., Koudokpon H., Fanou VBA., Dandjesso C. (2013). Etude ethnobotanique des plantes utilisées dans le traitement du diabète chez les femmes enceintes à Cotonou et Abomey-Calavi (Bénin). J. of Animal \& Plant Sci., 18 (1) : 2647 - 2658.

Gbekley EH., Karou DS., Gnoula C., Agbodeka K., Anani K., Tchacondo T., Agbonon A., Batawila K., Simpore J. (2015). Etude ethnobotanique des plantes utilisées dans le traitement du diabète dans la médecine traditionnelle de la région Maritime du Togo. Pan African Medical Journal, (20) : 437 - 452.
Gnagne AS., Camara D., Fofie NBY., Béné K., Ziribi GN. (2017). Etude ethnobotanique des plantes médicinales utilisées dans le traitement $\mathrm{du}$ diabète dans le Département de Zouénoula (Côte d'Ivoire). Journal of Appl. Biosci., (113): 11257 - 11266.

Houghton PJ., Raman A. (1998). Laboratory handbook for the fractionation of natural extracts, Ed Chapman and Hall, New York. 208 pages.

Kadri Y., Moussaoui A., Benmebarek A. (2018). Etude ethnobotanique de quelques plantes médicinales dans une région hyper aride du Sud-ouest Algérien « Cas du Touat dans la wilaya d'Adrar ». Journal of Animal \& Plant Sciences, 36 (2) : 5844 - 5857.

Kpetehoto WH., Johnson RC., Houeto EEM., Hounkpatin ASY., Boni G., Mignanwande ZF., Yedomonhan $\mathrm{H}$., Loko F. (2020). Ethnomedicinal study of Ocimum gratissimum Lin (Lamiaceae) in Cotonou. Journal of Complementary Medecine Research, JCMR- Volume., Numéro, 2020.

Kpètèhoto HW., Hessou S., Dougnon VT., Johnson RC., Boni G., Houéto EEM. (2017). Etude ethnobotanique, phytochimique et écotoxicologique de Ocimum gratissimum Linn (Lamiaceae) à Cotonou. Journal of Applied Biosciences, (109) : 10609 - 10617.

Lagnika L., Amoussa AMO., Adjileye AAR., Laleye A., Sanni A. (2016). Antimicrobial, antioxidant, toxicity and phytochemical assessment of extracts from Acmella uliginosa, a leaf-vegetable consumed in Bénin, West Africa. BMC Compl. and Alt. Med., 16 (34) : 1014 1023.

Lehout R., Maya L. (2015). Comparaison de trois méthodes d'extraction des composés phénoliques et des flavonoïdes à partir de la plante médicinale : Artemisia herba alba Asso. Mémoire de Master en Sciences de la Nature et de la Vie de 
l'Université des Frères Mentouri Constantine. 76 pages

Li HB., Cheng KW., Wong CC., Fan KW., Chen F., Jiang Y. (2007). Evaluation of antioxidant capacity and total phenolic content of different fractions of selected microalgae. Food Chem., 102 (3) : 771 6.

Mahmoudi S., Khali M., Mahmoudi N. (2013). Etude de l'extraction des composés phénoliques de différentes parties de la fleur d'artichaut (Cynara scolymus L.). Nat. \& Techno. B - Sci. Agro. et Biol., $2013,9: 35-40$.

Mehdioui R., Kahouadji A. (2007). Etude ethnobotanique auprès de la population riveraine de la forêt d'Amsittène : cas de la Commune d'Imi N'tlit (Province d'Essaouira). Section sciences de la vie, Rabat, Bul. de l'Inst. Sci., 2007, 29 : 11 20.

Mnayer D. (2014). Eco-Extraction des huiles essentielles et des arômes alimentaires en vue d'une application comme agents antioxydants et antimicrobiens. Thèse de Doctorat en science à l'Université d'Avignon. 157 pages

Nadhiya K., Vijayalakshmi K. (2014). Evaluation of total phenol, flavonoid contents and in vitro antioxidant activity of benincasa Hispida fruit extracts. Int. J. of Pharma. Chem. and Biol. Sci., 2014, 4 (2) : 332 338.

N'guessan K., Kadja B., Zirihi GN., Traoré D., Aké-Assi L. (2009). Screening phytochimique de quelques plantes médicinales ivoiriennes utilisées en pays Krobou (Côte-d'Ivoire). Sci. \& Nat., 2009, 6 (1) : 1 - 15.

Nounagnon, M.S., Dah-Nouvlessounon, D., N'tcha, C., Legba, B., Baba-Moussa, F., Adjanohoun, A., Baba-Moussa, L. (2018). Phytochemistry and biological activities of Crateva adansonii extracts. International Journal of Pharmacy and Pharmaceutical Sciences, 10, 62-67. http:/ /dx.doi.org/10.22159/ijpps.2018v 10i9.27197.

Olou BA., Bio A., Deleke Koko EIK., Djego

GJ., ET Sinsin AB. (2018).

Connaissances ethnobotaniques et

valorisation de deux plantes antihypertensives (Carissa edulis L. et Crateva adansonii DC) au Sud et au Centre du Bénin (Afrique de l'Ouest). Int. J. Biol. Chem. Sci. 12(6): 2602-2614. DOI: https://dx.doi.org/10.4314 /ijbcs .v12i6.11

Ramdane F. (2018). Contribution à l'étude des activités biologiques de quelques plantes médicinales du Sahara algérien : Nauplius graveolens, Ziriphus lotus et Capparis spinosa. Thèse de Doctorat en biologie. Universite Kasdi Merbah-Ouargla. 162 pages.

Saeed N., Muhammad R., Khan MS. (2012) Antioxidant activity, total phenolic and total flavonoid contents of whole plant extracts Torilis leptophylla L. BMC Complementary and Alternative Medicine 2012, 12:221 http:/ /www.biomedcentral.com/14726882/12/22

Tokoudagba J.M., Chabert P., Auger C., N'Gom S., Gbenou J., Moudachirou M., SchiniKerth V., Lobstein A. (2009). Recherche de plantes à potentialités antihypertensives dans la biodiversité béninoise. Journal Ethnopharmacologia, $n^{\circ} 44,10$ pages

Tsado NA., Lawal B., Santali ES., Mohammed AS., Balarabe M., Ibrahim HA., George JJ. (2015). Phytochemicals and Acute Toxicity Profile of Aqueous and Methanolic Extracts of Crateva adansonii Leaves in Swiss Albino Rats. Asian Journal of Biochemistry 10 (4): 173-179, 2015/ DOI: 10.3923/ajb.2015.173.179

Wagner, H. and Bladt, S. (2001) Plant Drugs Analisis, a Thin Layer Chromatography. 2nd Edition, Springer-Verlag Berlin, Heidelberg, New York 
WHO/MS-Bénin (2013). Renforcement du rôle de la médecine traditionnelle dans le système de santé au Benin. 47 pages.

Yémoa AL., Gbénou JD., Johnson RC., Djègo JG., Zinsou C., Moudachirou M., Quetin-Leclercq J., Bigot A., Portaels F. (2008). Identification et étude phytochimique de plantes utilisées dans le traitement traditionnel de l'ulcère de Buruli au Bénin. Ethnopharmacologia, $42: 48-55$. 